\title{
Chromosomal aneuploidy affects the global proteome equilibrium of colorectal cancer cells
}

\author{
Timo Gemoll ${ }^{\mathrm{a}, \mathrm{b}, \mathrm{c}, 1, *}$, Jens K. Habermann ${ }^{\mathrm{a}, \mathrm{c}, \mathrm{d}, 1, *}$, Susanne Becker ${ }^{\mathrm{b}}$, Silke Szymczak ${ }^{\mathrm{e}}$, \\ Madhvi B. Upender ${ }^{\mathrm{d}}$, Hans-Peter Bruch ${ }^{\mathrm{a}}$, Ulf Hellman ${ }^{\mathrm{f}}$, Thomas Ried ${ }^{\mathrm{d}}$, Gert Auer ${ }^{\mathrm{b}}$, Hans Jörnvall ${ }^{\mathrm{c}}$ \\ and Uwe J. Roblick ${ }^{\mathrm{a}, \mathrm{c}}$ \\ ${ }^{a}$ Section for Translational Surgical Oncology \& Biobanking, Department of Surgery, University of Lübeck, Lübeck, \\ Germany \\ ${ }^{\mathrm{b}}$ Karolinska Biomic Center, Karolinska Institutet, Stockholm, Sweden \\ ${ }^{\mathrm{c}}$ Department of Medical Biochemistry and Biophysics, Karolinska Institutet, Stockholm, Sweden \\ ${ }^{\mathrm{d}}$ Center for Cancer Research, National Cancer Institute, NIH, Bethesda, MD, USA \\ ${ }^{\mathrm{e}}$ Institute of Medical Biometry and Statistics, University of Lübeck, Lübeck, Germany \\ ${ }^{\mathrm{f}}$ Ludwig Institute for Cancer Research, Ltd. Uppsala, Uppsala, Sweden
}

\begin{abstract}
. cellular proteome equilibrium in general. found in colorectal cancer. Ingenuity Pathway Analysis (IPA). alterations involved in regulating genome stability.

\footnotetext{
${ }^{1}$ These authors contributed equally to this work.

${ }^{*}$ Corresponding author: Timo Gemoll, Ph.D., University of Lübeck, Ratzeburger Allee 160, D-23538 Lübeck, Germany. Tel.: +49 451 5003917; Fax: +49 451 5002069; E-mail: Gemoll@uniluebeck.de; Jens K. Habermann, M.D., Ph.D., University of Lübeck, Ratzeburger Allee 160, D-23538 Lübeck, Germany. Tel.: +49 451 5003336; Fax: +49 451 5002069; E-mail: Jens.Habermann@ chirurgie.uni-luebeck.de.
}

BACKGROUND: Chromosomal aneuploidy has been identified as a prognostic factor in the majority of sporadic carcinomas. However, it is not known how chromosomal aneuploidy affects chromosome-specific protein expression in particular, and the

OBJECTIVE: The aim was to detect chromosomal aneuploidy-associated expression changes in cell clones carrying trisomies

METHODS: We used microcell-mediated chromosomal transfer to generate three artificial trisomic cell clones of the karyotypically stable, diploid, yet mismatch-deficient, colorectal cancer cell line DLD1 - each of them harboring one extra copy of either chromosome 3, 7 or 13. Protein expression differences were assessed by two-dimensional gel electrophoresis and mass spectrometry, compared to whole-genome gene expression data, and evaluated by PANTHER classification system and

RESULTS: In total, 79 differentially expressed proteins were identified between the trisomic clones and the parental cell line. Up-regulation of PCNA and HMGB1 as well as down-regulation of IDH3A and PSMB3 were revealed as trisomy-associated

CONCLUSIONS: These results show that trisomies affect the expression of genes and proteins that are not necessarily located on the trisomic chromosome, but reflect a pathway-related alteration of the cellular equilibrium.

Keywords: Aneuploidy, genomic instability, mass spectrometry, pathway analysis, two-dimensional gel-electrophoresis

\section{Background}

Chromosomal aneuploidy is defined as any chromosome number that is not a multiple of the haploid complement [1]. It has been associated with severe 
developmental abnormalities in non-malignant diseases $[2,3]$ and is a defining feature of carcinomas $[4,5]$. For instance, one of the earliest chromosomal alterations observed in the development of sporadic colorectal carcinomas is a trisomy of chromosome 7 in adenomas [6]. This trisomy-7 is maintained during carcinogenesis and complemented by trisomies of chromosomes and chromosome arms 13, 8q, and 20, as well as losses of chromosomes 4 and 18 [7]. This pattern of chromosomal alterations is characteristic for colorectal cancer and also preserved in liver metastases of this cancer [8].

An analysis of colorectal cancer progression in 36 patients revealed a direct correlation of chromosomal aneuploidy with chromosome-specific gene expression levels, including trisomies of chromosomes 7 and 13 [8]. Chromosomal aneuploidy also affects expression levels of genes not located on the altered chromosome contributing to a massive dysregulation of the cellular transcriptome [8]. This observation was supported by separate introduction of chromosomes 3 , 7 , and 13 into a karyotypically diploid, yet mismatch repair-deficient colorectal cancer cell line. Regardless of chromosome or cell type, these chromosomal trisomies resulted in a significant increase in the average transcriptional activity of genes located on the trisomic chromosomes [9].

In general, with the implementation of current twodimensional gel-electrophoresis (2-DE) technologies, global protein analyses are possible [5, 10, 11]. Applying these techniques, it is feasible to establish an annotated protein expression map of cancer subtypes, which may elucidate the critical protein expression alterations responsible for the individual risk profile of cancer patients.

We now apply 2-DE technologies and mass spectrometry to detect chromosomal aneuploidyassociated expression changes in cell clones carrying trisomies found in colorectal cancer (Fig. 1).

\section{Methods}

\subsection{Microcell-mediated chromosome transfer}

Microcell-mediated chromosome transfer methodology was used as described [12, 13]. Cells were incubated for $48 \mathrm{~h}$ with $0.05 \mu \mathrm{g} / \mathrm{mL}$ Colcemid in media plus $20 \%$ serum to induce micronuclei formation.
They were centrifuged in the presence of $10 \mu \mathrm{g} / \mathrm{mL}$ cytochalasin B at $8000 \mathrm{rpm}$ for $1 \mathrm{~h}$ at $34^{\circ} \mathrm{C}$ to isolate micronuclei. Purified micronuclei were incubated with the recipient cells for 15 to $20 \mathrm{~min}$ in phytohemagglutinin P containing medium $(100 \mu \mathrm{g} / \mathrm{mL})$. Clones were expanded and tested for incorporation of neomycin-tagged chromosomes by fluorescence in situ hybridization (FISH) using whole chromosomespecific paint probes and a neomycin-specific DNA probe. We generated three DLD1-derivative cell lines: DLD1+3 containing an extra copy of chromosome 3, DLD1+7 containing an extra copy of chromosome 7, and DLD1+13 containing an extra copy of chromosome 13 (Additional file 1).

\subsection{Genomic and transcriptomic analysis}

Samples processed for protein expression were also analyzed by FISH, spectral karyotyping (SKY), and gene expression. A detailed description of genomic and transcriptomic sample processing, analysis, and evaluation has been published earlier by Upender et al. [9].

\subsection{Protein quantification}

Protein concentrations of samples were determined in quadruplicates using 96-well microplates by addition of $25 \mu \mathrm{L}$ concentrated assay reagent (BioRad) to $1 \mu \mathrm{L}$ solubilized sample diluted in $100 \mu \mathrm{L}$ Milli-Q water [14]. The plates were read in a Multiscan reader (Labsystems).

\subsection{Two-dimensional gel electrophoresis}

$75 \mu \mathrm{g}$ of the derived clones were mixed with isoelectric focusing (IEF) solvent (7 M urea, $2 \mathrm{M}$ thiourea, $100 \mathrm{mM}$ DTT, $1 \%$ 3-[3-chloramidopropyl]dimethylammonio-1-propanesulfonate [CHAPS], $0.5 \%$ immobilized $\mathrm{pH}$ gradient [IPG] buffer, $0.3 \%$ dithiothreitol [DTT] and a trace of bromophenol blue) to a final volume of $300 \mu \mathrm{L}$. This mixture was then applied to two micro range ReadyStrips by cup-loading (17 cm, pH $4.7-5.9$ and $\mathrm{pH} 5.5-6.7$, respectively; Bio-Rad) and actively rehydrated at $50 \mathrm{~V}$, $20^{\circ} \mathrm{C}$ for $16 \mathrm{~h}$. Proteins were isoelectrically focused stepwise, starting at $500 \mathrm{~V}$ and increasing to $8000 \mathrm{~V}$ to reach a total of $52.900 \mathrm{Vh} /$ gel. Ready Strips were then stored at $-80^{\circ} \mathrm{C}$ until second dimension processing. After isoelectric focusing, the strips were equilibrated 
Chromosomal aneuploidy affects the global proteome equilibrium of colorectal cancer cells

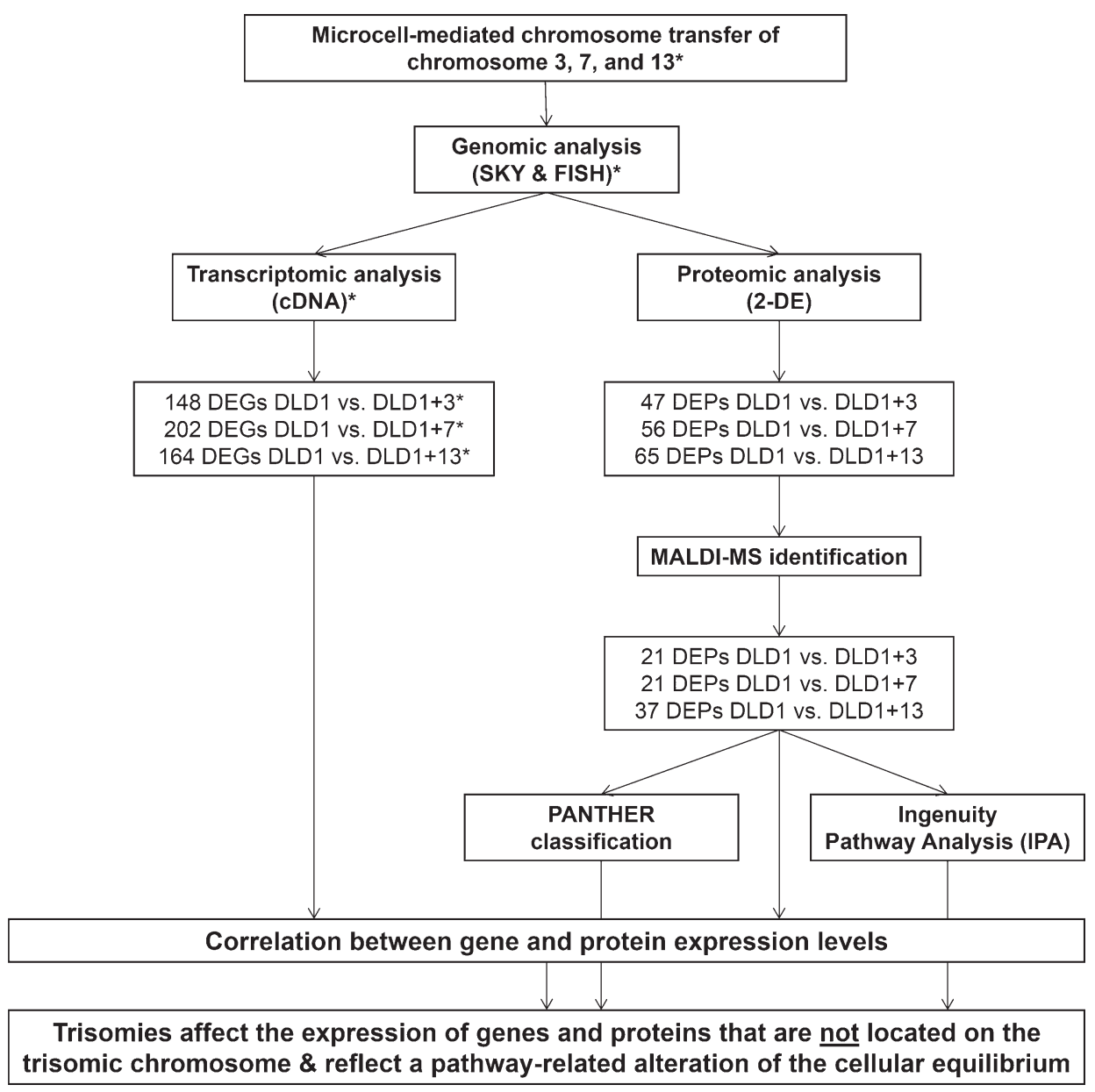

Fig. 1. Technical workflow of the study design for comperative proteomic analysis. *published by Upender et al. in 2004. SKY, Spectral karyotyping; FISH, Fluorescence in-situ hybridization; 2-DE, Two-dimensional gel electrophoresis; DEG, Differential expressed gene; DEP, Differential expressed protein.

for $2 \times 15$ min with $50 \mathrm{mM}$ Tris- $\mathrm{HCl}$, $\mathrm{pH} 8.8$, in $6 \mathrm{M}$ urea, $30 \%$ glycerol and $2 \%$ SDS. DTT $(2 \%)$ was included in the first and iodoacetamide $(2.5 \%)$ in the second equilibration step to reduce and alkylate free thiols. $10-13 \%$ linear acrylamide gradient gels $(1.5 \times 200 \times 230 \mathrm{~mm})$ with $1 \%$ SDS were used for second-dimension gel electrophoresis. The IPG strip was placed on the surface of the second-dimension gel, and sealed with $0.5 \%$ agarose in SDS-electrophoresis buffer $(25 \mathrm{mM}$ Tris base, $192 \mathrm{mM}$ glycine, $0.1 \%$ SDS). The gels were run overnight at $100 \mathrm{~V}, 12^{\circ} \mathrm{C}$. Gels were stained with silver nitrate, scanned with a flatbed densitometer (GS 710, Bio-Rad, CA, USA), and analysed with the PDQuest software (BioRad, CA, USA, version 8.0.1).

\subsection{Statistics}

2-DE expression data were pre-processed by $\log$ transformation. In order to measure agreement between replications, Bland-Altman plots were then computed and proteins with expression value differences outside of the limits of agreement were excluded from further analysis [15]. Comparisons of expression 
values of proteins between the modified cell lines and the reference were performed using the student's $t$ tests with a significance level of 0.05 . $P$-values were not adjusted for multiple testing. Fold changes were calculated as exponentiated mean differences. The statistical software package $\mathrm{R}$ version 2.6.1 was used for all statistical analyses.

\subsection{In-gel digestion and mass spectrometry (MALDI-ToF)}

Spots from 2-DE gels were manually cut with a scalpel in a laminar air-flow bench. After destaining followed by digestion with trypsin as described [16] (Additional file 2), the fragments were analyzed by matrix assisted laser desorption/ionization time of flight mass spectrometry (MALDI-ToF-MS) in an Ultraflex III TOF/TOF instrument (Bruker Daltonics, Bremen, Germany).

\subsection{Functional evaluation and pathway analysis}

Theoretical detectability of proteins from all artificially inserted chromosomes was evaluated by an extensive Entrez-Gene and ProteinAtlas ${ }^{\circledR}$ database search. All genes on chromosomes 7 and 13 were evaluated for their detectability in our 2-DE approach $(\mathrm{pH}$ 4.7 to 6.7 ; MW 10 to $200 \mathrm{kDa}$ ). Chromosome 3 was not considered, mainly since its insertion served as a positive control while not being gained during colorectal cancer development. Each group comparison was used to evaluate the impact of chromosomal aneuploidy on the global protein expression. Functional analysis of all identified proteins was carried out using the PANTHER classification system (www.pantherdb.org/).

Identified proteins were analyzed by the Ingenuity pathway analysis software (Ingenuity IPA 9.0 Build 3211) to evaluate chromosomal aneuploidy-associated proteome changes. The Ingenuity pathway analysis program uses a knowledge-base derived from the literature to relate gene and protein products based on their interaction and function. This software is designed to identify dynamically generated biological networks, global canonical pathways, and global functions. A score reflects the negative logarithm of the $\mathrm{P}$ value, which indicates the likelihood of the focus-proteins in a network being found together by random chance. A score higher than five was set as the threshold to determine a valid network.

\section{Results}

\subsection{Microcell-mediated chromosome-transfer and genome analysis}

SKY was performed to determine whether chromosome transfer was successful, and whether it induced secondary karyotypic changes. Chromosome-specific FISH probes assessed the efficiency of maintaining extra copies of chromosomes 3, 7, and 13 under neomycin-selective cell culture conditions. Both analyses proved that all three derivative clones (DLD1+3, DLD1+7, DLD1+13) maintained the diploid karyotype of the parental cell line, contained the additional copy of the introduced chromosome in over $80 \%$ of cells analyzed, and showed no secondary karyotypic changes except for a loss of the Y chromosome in all DLD1+3 and in a small percentage of DLD1+7 cells [9].

\subsection{Transcriptomic profiling}

The influence of chromosomal aneuploidy on the RNA expression level was examined by considering only those genes whose expression ratios were $>2.0$ (up-regulated) or $<0.5$ (down-regulated) when compared to the parental cell line. Gene expression data have been published earlier by Upender et al. [9].

For DLD1+3, an extra copy of chromosome 3 changed expression levels for 148 genes throughout the entire genome: 81 genes were up- and 67 genes down-regulated. However, only 17 of these affected genes $(11.5 \%)$ mapped to chromosome 3. Notably, all 17 genes were up-regulated and no gene on chromosome 3 was found to be down-regulated. For DLD1+7, expression levels of 202 genes were found to be affected: 155 genes were up- and 47 down-regulated. While 35 of these $(17.3 \%)$ mapped to chromosome 7, 32 were up- and three down-regulated. Regarding DLD1+13, overall 164 genes were found to be differentially expressed: 92 up- and 72 down-regulated. Of these, ten genes (6.1\%) mapped to chromosome 13, all being up-regulated [9]. Strikingly, none of the genes were affected in common among any of the three cell line clones.

\subsection{Two-dimensional gel electrophoresis and pathway analysis}

2-DE analysis revealed overall 168 spots corresponding to proteins that were differentially 
Table 1

Identifications of the DLD1 versus DLD1+7 analysis

\begin{tabular}{|c|c|c|c|c|}
\hline Sample ID * & Protein Identity & Chromosome & $\mathrm{Fc}$ & Sequence coverage [\%] \\
\hline \multicolumn{5}{|c|}{ Lower expression in DLD1+7 cell clone } \\
\hline 1215 & EEF1D: Eukar. translation elongation factor & $8 q 24.3$ & 0.81 & 40 \\
\hline 1220 & TXNDC5: Thioredoxin domain containing 5 & $6 \mathrm{p} 24.3$ & 0.46 & 20 \\
\hline 1613 & TCP1: T-complex 1 & $6 \mathrm{q} 25.3-\mathrm{q} 26$ & 0.55 & 29 \\
\hline 3116 & DCI: Dodecenoyl-Coenzyme A delta isomerase & $16 \mathrm{p} 13.3$ & 0.81 & 20 \\
\hline 3314 & DNAJB11: DnaJ (Hsp40) homolog, member 11 & $3 q 27.3$ & 0.38 & 28 \\
\hline 4104 & CTSD: Cathepsin D & $11 \mathrm{p} 15.5$ & 0.47 & 27 \\
\hline 4104 & PSMB3: Proteasome subunit & $17 \mathrm{q} 12$ & 0.53 & 42 \\
\hline 5003 & APRT: Adenin phosphoribosyltransferase & $16 \mathrm{q} 24$ & 0.80 & 45 \\
\hline 5012 & ADI1: Acireductone Dioxygenase 1 & $2 \mathrm{p} 25.3$ & 0.74 & 32 \\
\hline 5301 & EIF3I: Eukar. Translation initiation factor & $1 \mathrm{p} 34.1$ & 0.67 & 44 \\
\hline 6210 & STUB1: STIP1 homolgy and U-box containing protein 1 & $16 \mathrm{p} 13.3$ & 0.87 & 20 \\
\hline 7303 & IDH3A: Isocitrate dehydrogenase 3 alpha & $15 \mathrm{q} 25.1$ & 0.71 & 21 \\
\hline 7304 & AKR1A1: Aldo-keto reductase family 1, member A1 & $1 \mathrm{p} 33-\mathrm{p} 32$ & 0.56 & 21 \\
\hline \multicolumn{5}{|c|}{ Higher expression in DLD1+7 cell clone } \\
\hline 111 & YWHAZ: 14-3-3 & $8 \mathrm{q} 23.1$ & 3.31 & 47 \\
\hline 2203 & TXNL1: Thioredoxin-like 1 & $18 \mathrm{q} 21.31$ & 1.29 & 37 \\
\hline 4220 & PSMA1: Proteasome & $11 \mathrm{p} 15.1$ & 2.73 & 13 \\
\hline 5016 & CFL1: Cofilin 1 & $11 \mathrm{q} 13$ & 1.78 & 40 \\
\hline 5128 & HMGB1: High-mobility group box 1 & $13 q 12$ & 2.01 & 26 \\
\hline 6212 & PDXK: Pyridoxal kinase & $21 \mathrm{q} 22.3$ & 1.60 & 25 \\
\hline 6620 & EPB41: Erythrocyte membrane protein band 4.1 & $1 \mathrm{p} 33-\mathrm{p} 32$ & 1.28 & 9 \\
\hline 7102 & PSME1: Proteasom activator subunit 1 isoform 1 & $14 \mathrm{q} 11.2$ & 1.23 & 48 \\
\hline
\end{tabular}

Fc, Fold change. *Due to two different match-sets (low- and high $\mathrm{pH}$ range) double sample IDs are possible.

expressed between the trisomic and parental cell lines $(p<0.05)$.

The introduction of an extra copy of chromosome 3 resulted in differential regulation of 47 protein spots, of which $21(47 \%)$ could be identified by mass spectrometry: ten were up- and eleven down-regulated and none of these proteins mapped to chromosome 3 (Additional file 3). These proteins were found to constitute two networks: one was associated with Lipid Metabolism, Molecular Transport, and Small Molecule Biochemistry, the other one (score of 13) with Cellular Development, Cellular Growth and Proliferation and Reproductive Development and Function (Additional file 4). YWHAZ and MYC are central nodes of these networks, and are associated with diseases and functions related to Cancer, Development Disorder, and Genetic Disorder $(p<0.00001$ to $p<0.0410)$ (Additional file 5).

For clone DLD1+7, 56 protein spots were differentially regulated and 21 were identified $(37.5 \%)$ : eight being higher- and 13 lower-expressed. Of these proteins, no protein mapped to chromosome 7 (Table 1). Pathway analysis yielded two overlapping networks (scores of 29 and 18). The highest-ranked network was associated with Cellular Assembly and Organization, Post-Translational Modification, and Skeletal and Muscular Disorders. This network interacts via PSMB10 with the second network which is associated with Antigen Presentation, Cell-To-Cell Signalling and Interaction, and Haematological System Development and Function. Furthermore, Cellular Assembly and Organization, Cell Death, and Cell Morphology $(p<0.0472)$, Cardiovascular Disease $(p<0.030)$, Haematological Disease $(p<0.0207)$, and Inflammatory Response $(p<0.0434)$ are distinct molecular functions and diseases of these networks (Additional file 4 and Fig. 2a).

Regarding DLD1+13, 65 differentially expressed protein spots were detected and $37(57 \%)$ were identified: 14 being up- and 23 down-regulated. None of the identified proteins mapped to chromosome 13 (Table 2). The proteins were involved in three different networks, one (score 24) being associated with Cell Cycle, Cellular Movement, DNA Replication, Recombination, and Repair with nuclear factor of kappa light polypeptide gene enhancer in B-cells $(\mathrm{Nf} \kappa \mathrm{B})$ and proliferating cell nuclear antigen (PCNA) as central nodes. Another network (score 23) correlated 
a)

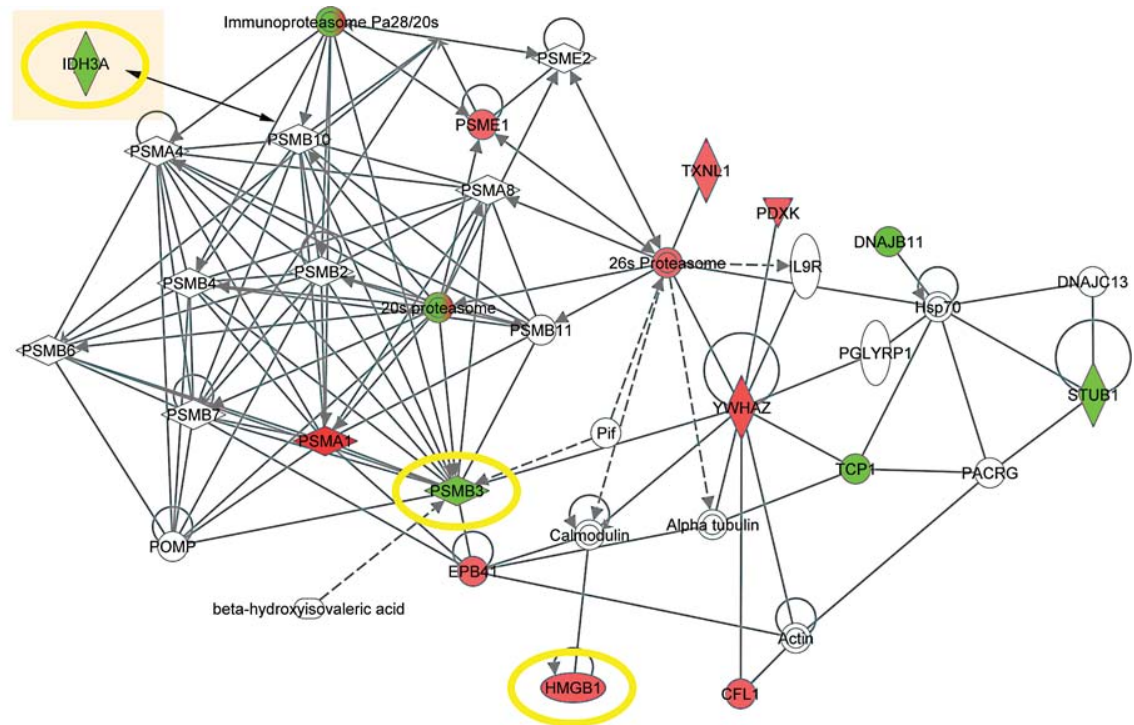

b)

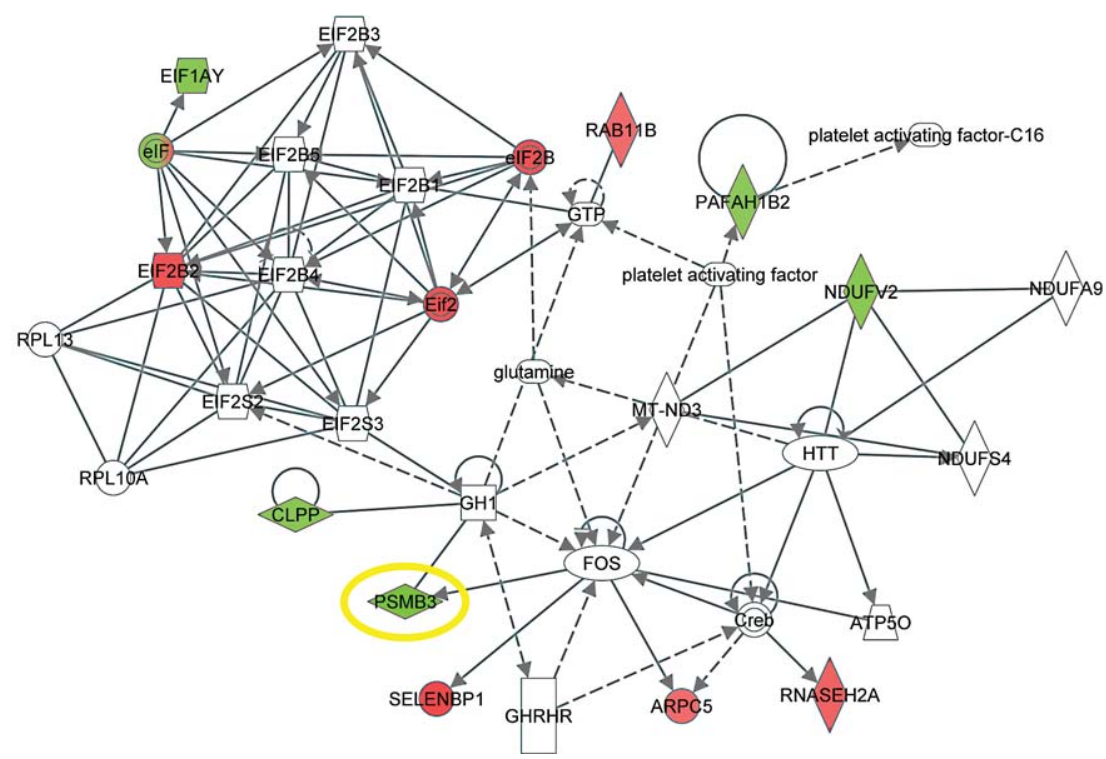

Fig. 2. IPA-based pathway analysis of differential expressed proteins. (a) IPA gene pathway associated with Cellular Assembly and Organization containing PSMB3 and HMGB1 as significant regulated proteins in the DLD1+7 comparison. This network overlaps with network 2 (underlaid in orange) associated with Antigen Presentation containing IDH3A. Red and green designations indicate over- and under-expressed proteins in the respective groups. PSMB3, HMGB1, and IDH3A are highlighted in yellow. (b) IPA gene pathway associated with Developmental Disorder, Nucleic Acid Metabolism, and Genetic Disorder containing PSMB3 as a significant down- regulated gene and protein in the DLD1+13 comparison. Red and green designations indicate over- and under-expressed proteins in the respective groups. PSMB3 is highlighted in yellow.

with Cellular Growth and Proliferation, Nucleic Acid Metabolism, and Small Molecule Biochemistry. The third network (score 21) was associated with Development Disorder, Gene Expression, and Genetic Disorder. All three networks showed diseases and functions related to Cancer, Genetic Disorder, and
Inflammatory Disease $(p<0.00001$ to $p<0.0416)$ and Post-Translational Modification, Protein Folding, and Protein Synthesis $(p<0.00001$ to $p<0.0438)$ (Additional file 4 and Fig. 2b).

Two proteins, isocitrate dehydrogenase $3 \alpha$ (IDH3A) and proteasome subunit beta type 3 
Table 2

Identifications of the DLD1 versus DLD1+13 analysis

\begin{tabular}{|c|c|c|c|c|}
\hline Sample ID * & Protein Identity & Chromosome & $\mathrm{Fc}$ & Sequence coverage [\%] \\
\hline \multicolumn{5}{|c|}{ Lower expression in DLD1+13 cell clone } \\
\hline 1220 & COPE: Coatomer protein complex & $19 \mathrm{p} 13.11$ & 0.43 & 11 \\
\hline 1613 & WARS: Tryptophanyl-tRNA synthetase & $14 q 32.31$ & 0.46 & 19 \\
\hline 3005 & EIF1AY: Eukaryotic translation initiation factor 1A, Y-linked & Yq11.222 & 0.80 & 36 \\
\hline 4104 & PSMB3: Proteasome (prosome, macropain) subunit & $17 \mathrm{q} 12$ & 0.28 & 27 \\
\hline 4109 & NT5C: 5', 3'-nucleotidase, cytosolic & $17 \mathrm{q} 25.1$ & 0.59 & 15 \\
\hline 4308 & CAMKV: CaM kinase-like vesicle-associated & $3 \mathrm{p} 21.31$ & 0.64 & 18 \\
\hline 4420 & PSMD11: Proteasome (prosome, macropain) 26 S subunit & $17 \mathrm{q} 11.2$ & 0.57 & 14 \\
\hline 4510 & HSPD1: Heat shock $60 \mathrm{kDa}$ protein 1 & $2 \mathrm{q} 33.1$ & 0.41 & 16 \\
\hline 5106 & PSPH: Phosphoserine phosphatase & $7 \mathrm{p} 15.2-\mathrm{p} 15.1$ & 0.65 & 20 \\
\hline 5114 & HSPB1: Heat shock $27 \mathrm{kDa}$ protein 1 & $7 q 11.23$ & 0.37 & 43 \\
\hline 5115 & PAFAH1B2: platelet-activating factor acetylhydrolase, isoform Ib & $11 \mathrm{q} 23$ & 0.88 & 16 \\
\hline 5323 & MPST: Mercaptopyruvate sulfurtransferase & $22 q 13.1$ & 0.53 & 35 \\
\hline 5423 & ACTB: Actin, beta & $7 \mathrm{p} 15-\mathrm{p} 12$ & 0.16 & 18 \\
\hline 6105 & CLPP: ClpP caseinolytic peptidase & $19 \mathrm{p} 13.3$ & 0.77 & 19 \\
\hline 6401 & RUVBL1: RuvB-like 1 (E. coli) & $3 q 21$ & 0.50 & 23 \\
\hline 7019 & UBE2N: Ubiquitin-conjugating enzyme E2N & $12 \mathrm{q} 22$ & 0.58 & 55 \\
\hline 7104 & POLR2E: Polymerase (RNA) II & $19 \mathrm{p} 13.3$ & 0.93 & 30 \\
\hline 7114 & GRB2: Growth factor receptor-bound protein 2 & $17 q 24-q 25$ & 0.79 & 26 \\
\hline 7117 & NDUFV2: NADH dehydrogenase flavoprotein 2 & 18p11.31-p11.2 & 0.73 & 23 \\
\hline 7303 & IDH3A: Isocitrate dehydrogenase 3 (NAD+) $\alpha$ & $15 \mathrm{q} 25.1-\mathrm{q} 25.2$ & 0.69 & 23 \\
\hline 7308 & UROD: Uroporphyrinogen decarboxylase & $1 \mathrm{p} 34$ & 0.74 & 22 \\
\hline 8421 & DNAJA2: DnaJ (Hsp40) homolog & $16 \mathrm{q} 12.1$ & 0.68 & 12 \\
\hline 9305 & AKNA: AT-hook transcription factor & $9 q 32$ & 0.60 & 7 \\
\hline \multicolumn{5}{|c|}{ Higher expression in DLD $1+13$ cell clone } \\
\hline 28 & MRCL3: Myosin regulatory light chain MRCL3 & $18 \mathrm{p} 11.31$ & 4.37 & 18 \\
\hline 235 & PCNA: Proliferating cell nuclear antigen & 20pter-p12 & 1.24 & 39 \\
\hline 1613 & UBQLN1: Ubiquilin 1 & $9 \mathrm{q} 21.2-\mathrm{q} 21.3$ & 1.26 & 20 \\
\hline 2112 & CTSD: Cathepsin D & $11 \mathrm{p} 15.5$ & 1.67 & 23 \\
\hline 2214 & RNASEH2A: Ribonuclease H2, subunit A & $19 \mathrm{p} 13.13$ & 1.57 & 15 \\
\hline 2215 & EFHD2: EF-hand domain family, member D2 & $1 \mathrm{p} 36.21$ & 1.76 & 27 \\
\hline 5017 & ARPC5: Actin related protein $2 / 3$ complex, subunit 5 & $1 \mathrm{q} 25.3$ & 1.15 & 29 \\
\hline 5018 & EIF2B2: Eukaryotic translation initiation factor 2B, subunit 2 & $14 \mathrm{q} 24.3$ & 2.14 & 13 \\
\hline 6406 & TUFM: Tu translation elongation factor, mitochondrial & $16 \mathrm{p} 11.2$ & 6.50 & 42 \\
\hline 6507 & PDIA3: Protein disulfide isomerase family A & $15 q 15$ & 1.45 & 39 \\
\hline 7319 & CAPG: Capping protein (actin filament) & $2 \mathrm{p} 11.2$ & 2.31 & 13 \\
\hline 7403 & $\begin{array}{l}\text { BRF1: BRF1 homolog, subunit of RNA polymerase III } \\
\text { transcription initiation factor IIIB }\end{array}$ & $14 \mathrm{q}$ & 2.21 & 5 \\
\hline 8114 & RAB11B: RAB11B, member RAS oncogene family & $19 \mathrm{p} 13.2$ & 1.22 & 35 \\
\hline 8419 & SELENBP1: Selenium binding protein 1 & $1 q 21-q 22$ & 4.22 & 57 \\
\hline
\end{tabular}

Fc, Fold change. *Due to two different match-sets (low- and high pH range) double sample IDs are possible.

(PSMB3), were found to overlap between all group comparisons and are significantly down-regulated in both, the DLD1+7 and DLD1+13 comparison.

\subsection{Function and clinical relevance of identified proteins}

In addition to evaluate pathway and network interactions of the identified proteins, we retrieved their molecular functions using the PANTHER classification system [17]. For DLD1+3, eight identified proteins showed molecular functions for catalytic activity $(35 \%)$ or binding $(35 \%)$, respectively. DLD $1+7$ revealed proteins mainly interacting in catalytic activity $(n=10 ; 53 \%)$, binding $(n=4 ; 21 \%)$, and translation regulatory activity $(n=2 ; 11 \%)$, while DLD1+13 revealed proteins of catalytic activity $(n=17 ; 39 \%)$, binding $(n=14 ; 32 \%)$, and structural molecule activity $(n=6 ; 14 \%)$ (Fig. 3). The largest functional groups 
a)

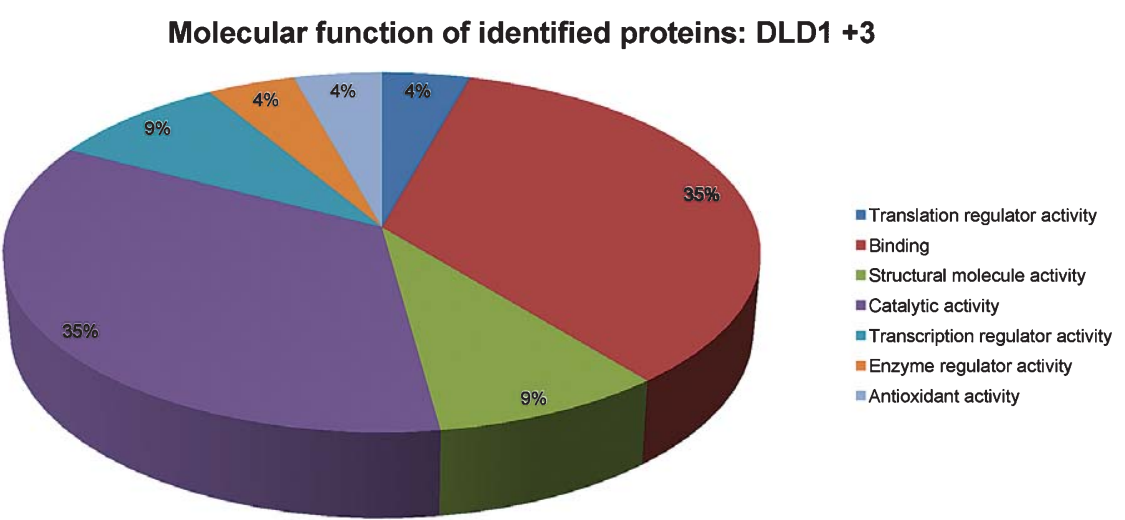

b) Molecular function of identified proteins: DLD1 +7

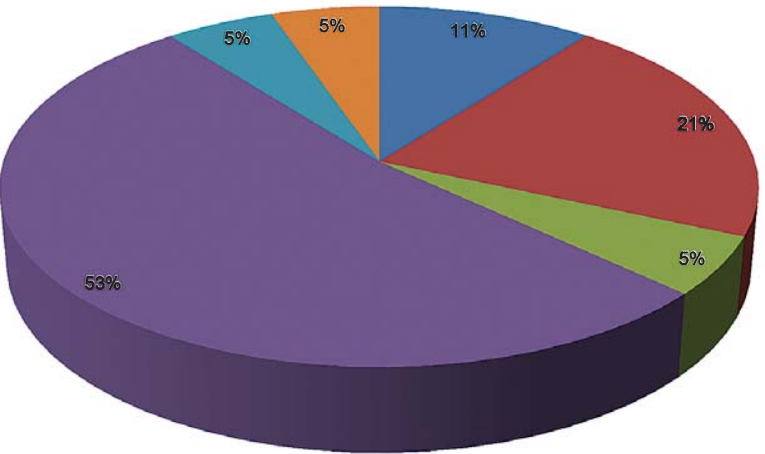

- Translation regulator activity ainding

- Structural molecule activity - Catalytic activity - Transcription regulator activity = Ion channel activity

c)

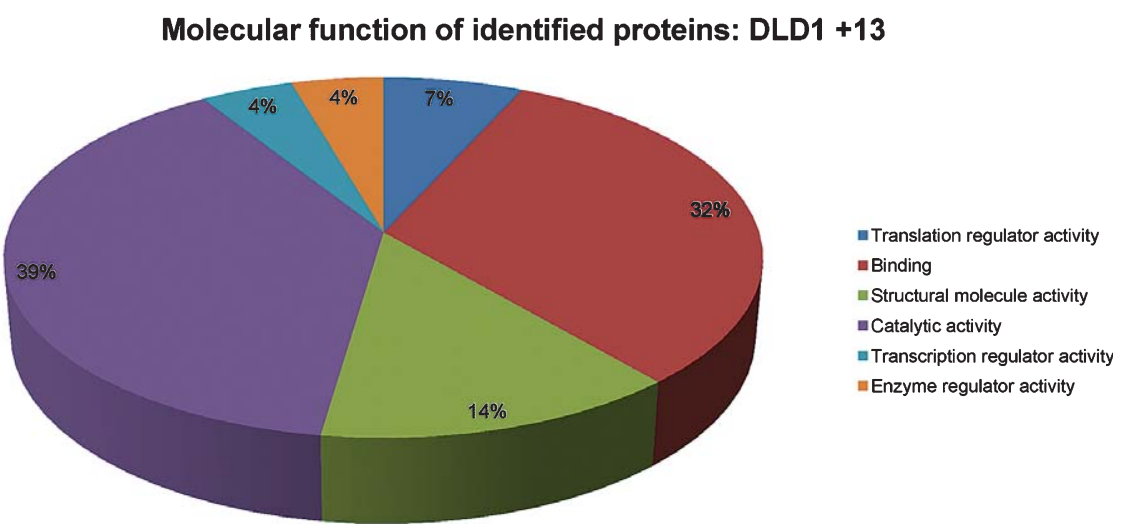

Fig. 3. Molecular functions of identified proteins in the DLD1+3 (a), DLD1+7 (b), DLD1+13 (c) comparisons based on the PANTHER classification system.

in all group comparisons were catalytic activity and binding for which DLD1+3 and DLD1+13 display a more similar pattern of molecular functions than DLD+7. Particularly proliferating cell nuclear antigen (PCNA) and high-mobility group box 1 (HMGB1) show DNA binding characteristics and also play a central role in the detected high-ranked networks of DLD1+7 and DLD1+13. Interestingly, the introduc- tion of trisomy 7 , the earliest detectable chromosomal alteration at pre-malignant stages of colorectal tumors, showed a substantially decreased function for structural molecule activity but an increased function for catalytic activity and translational regulatory activity compared to trisomies of chromosome 3 and 13. Furthermore, only DLD+7 showed functions regarding ion channel activity. 
Table 3

Summary of gene/protein correlation

\begin{tabular}{|c|c|c|c|c|}
\hline & \multicolumn{2}{|c|}{ DLD1+7 } & \multicolumn{2}{|c|}{ DLD1+13 } \\
\hline & Genes & Proteins & Genes & Proteins \\
\hline $\begin{array}{l}\text { No. of genes (coding and non-coding) and theoretical detectable proteins } \\
\text { by } 2-\mathrm{DE}^{*} \text { on artificial chromosome }\end{array}$ & 988 & 297 & 353 & 113 \\
\hline No. of differentially expressed genes $\# /$ proteins & $202(20.4 \%)$ & $56(18.9 \%)$ & $164(46.4 \%)$ & $65(57.5 \%)$ \\
\hline No. of identified proteins & & $21(37.5 \%)$ & & $37(56.9 \%)$ \\
\hline $\begin{array}{l}\text { No. of differentially expressed genes/proteins located on artificial } \\
\text { chromosome }^{\#}\end{array}$ & $35(3.54 \%)$ & $0(0 \%)$ & $10(2.83 \%)$ & $0(0 \%)$ \\
\hline $\begin{array}{l}\text { No. of theoretically detectable proteins in respect to the percentage of } \\
\text { differentially expressed genes located on artificial chromosome }\end{array}$ & & $10(3.37 \%)$ & & $3(2.65 \%)$ \\
\hline
\end{tabular}

*pH 4.7 to 6.7 ; MW 10 to $200 \mathrm{kDa}$. ${ }^{\#}$ Upender et al. [9].

\subsection{Correlation between gene and protein expression levels}

For 40 of all 79 identified proteins, the corresponding cDNA was included in our microarray platform. Out of those differentially expressed proteins, one corresponding gene, PSMB3 (located on chromosome 17q12), showed a significant down-regulation of both, the mRNA and protein expression level. Regarding the latter, PSMB3 is significantly downregulated in the DLD1+7 and DLD1+13 comparisons. PSMB3 is, however, not encoded by a gene located on one of the trisomic chromosomes. Interestingly, none of the identified proteins mapped to either of the trisomic chromosomes. We therefore evaluated the theoretical protein detectability by our 2-DE approach (pH 4.7 to 6.7 ; MW 10 to $200 \mathrm{kDa}$ ). This was performed for all coding genes located on the trisomic chromosomes 7 and 13 by a detailed ProteinAtlas (www.proteinatlas.org; 2010-07-22) and Entrez-Gene (www.ncbi.nlm.nih.gov/gene; 2010-07-22) search. Based on the total number of coding and noncoding genes of each inserted artificial chromosome all translated proteins were specified by their individual isoelectric point and molecular mass (Table 3).

Regarding chromosome 7, we identified 988 coding and non-coding genes, of which 297 proteins could have been detected with our 2-DE approach. In comparison, we detected 35 of the 988 coding genes (3.54\%) to be differentially expressed and located on chromosome 7 . If the same percentage of differentially expressed genes (DEGs) would be applicable for the detection of differentially expressed proteins (DEPs), only ten out of 297 (3.54\% of 297) theoretically detectable proteins would have been expected to be detected by our 2-DE analysis (Table 3 ).
Regarding chromosome 13, we identified 353 coding and non-coding genes, from which 113 proteins are theoretically detectable by our 2-DE approach. cDNA expression analysis revealed ten DEGs $(2.83 \%)$ that mapped to chromosome 13 . Computing the percentage of DEGs of chromosome 13 to the protein level revealed that only three out of 113 (2.83\% of 113$)$ theoretically detectable proteins would have been expected (Table 3).

Additionally, a subsequent evaluation of all pathway-interacting proteins for their chromosomal location was carried out. Notably, only two proteins, glycyl-tRNA synthetase (GARS) in the DLD1+7 analysis and adenylate cyclase-associated protein 2 (CAP2) in the DLD1+13 analysis revealed then a chromosomal locus on the artificial chromosome.

\section{Discussion}

In order to analyze how chromosomal aneuploidies affect protein expression levels, we now generated an experimental model system in which the only genetic alteration between parental and derived cell line is an extra copy of a single chromosome. Based on our present results with this system, several important conclusions regarding the impact of chromosomal aneuploidy on the cellular equilibrium can be drawn:

At the transcriptional level, we detected a substantial correlation between the artificially induced chromosome and the expression levels of genes located on that particular chromosome. These results were published earlier by Upender et al. [9]. Interestingly, Sengupta et al. performed 3D-FISH analysis by confocal laser scanning microscopy and 3D distance measurements and observed that the artificially introduced human 
chromosomes maintained a position similar to their endogenous homologues in the 3D interphase nucleus. These findings would be consistent with the interpretation that chromosome-wide transcription requires a conserved nuclear position in DLD-1 colorectal cancer cells [18]. Further evaluation of the effects of trisomies on the gene expression levels revealed that only $5-20 \%$ of the dysregulated genes were related to the trisomic chromosome, while the remaining DEGs were located elsewhere. Therefore, in addition to trisomyspecific transcriptional dysregulation, we observed trans-effects on global gene expression changes [9].

At the translational level, our analysis detected only one protein, proteasome subunit beta type 3 (PSMB3; located at 17q12), that showed a significant down-regulation equivalent to the transcriptional regulation in the DLD1+13 clone. The minor overlap between gene- and protein expression data might originate from the fact that - under genome-stable or genome-unstable conditions - translation of RNA can be modified in multiple manners: $\mathrm{N}$-glycosylation, phosphorylation, acetylation, and ubiquination are responsible for inferring the activity of the proteins in enzymatic reactions and signaling pathways [19, 20]. Indeed, our pathway analysis of the identified proteins dissected molecular and cellular functions that are specific for post-translational modifications (Additional file 4). Furthermore, the theoretical detectability by our 2-DE approach of proteins translated by the inserted chromosomes revealed only a limited number of potentially differentially expressed proteins. Interestingly, none of the 58 identified proteins of the DLD1+7 and DLD1+13 clones mapped to the trisomic chromosome even though ten (DLD+7) and three (DLD1+13) DEPs were theoretically expected to do so (Table 3). However, the ratio of differentially expressed genes and proteins is strikingly similar in DLD1+7 $(0.20$ versus 0.19 ) and DLD1+13 (0.46 versus 0.58 ). These findings are in line with studies of Stingele et al. and Torres et al. that show a lower abundance of proteins coded on the extra chromosomes in human cell lines and a partial compensation of the abundance of protein complexes in aneuploid yeast cells, respectively [21, 22].

In addition to all DEPs we also evaluated the pathway-interacting proteins for their chromosomal location: we could detect GARS (DLD1+7) and CAP2 (DLD1+13) to map to the introduced trisomies. The weak correlation between the transcriptional dysregulation induced by artificially introduced chromosomes and protein expression levels located on that particu- lar chromosome strongly suggests that trisomies affect protein expression globally rather than being restricted to genes/proteins located on the trisomic chromosome. This phenomenon is consistent with the fact that genes of a certain pathway are not located on a single chromosome but widely spread throughout the entire genome. One has to consider though that 2-DE allows analysis of a subset of the overall proteome, but there is still no technology that matches 2-DE in its ability for parallel expression profiling of large sets of complex protein mixtures. Beneficially, 2-DE delivers a map of intact proteins which reflects changes in protein expression levels, isoforms, post-translational modifications and sample quality (Additional file 6).

Most of the proteins identified here are dominated by molecular functions of catalytic activity and binding. We found two such proteins being up-regulated: proliferating cell nuclear antigen (PCNA, chromosomal location at 20pter-p12) and high-mobility group box 1 (HMGB1, chromosomal location at 13q12). Both proteins not only show DNA binding characteristics but also play a central role in the high-ranked networks specific for DLD1+7 and DLD1+13 that were amongst others associated with DNA and RNA metabolism as reported earlier [21]. The PCNA protein is a replication accessory factor found in the nucleus. PCNA forms a homo-trimeric ring around the DNA that serves as a binding platform for DNA polymerases. In doing so, PCNA enhances the processing activity of DNA polymerases [23, 24]. PCNA also modulates the fidelity of DNA synthesis in vitro [25] and interacts with factors involved in nucleotide excision repair [26], mismatch repair (MMR) [27, 28], and base excision repair [29]. PCNA further participates in the processing of branched DNA structures, including those formed during lagging-strand DNA synthesis [30]. The abundance of PCNA-interacting proteins led to the suggestion that PCNA may serve as a docking molecule for many proteins and coordinate various aspects of DNA synthesis and repair [31]. Of the above-mentioned processes, DNA polymerase fidelity, MMR, and branch structure processing are mutation-suppressing mechanisms that need to be tightly regulated and coordinated to ensure proper chromosome replication and integrity [32]. Thus, with an up-regulation of the PCNA expression in the DLD+13 clones, the cells seem to actively counteract trisomy-associated genomic instability. HMGB1 is a non-histone chromosomal structural protein that binds to non-B type DNA participating in multiple processes such as transcription, replication recombination, 
DNA repair and genomic stability [33]. HMGB1 is over-expressed in most human tumors, including breast carcinomas, melanomas, gastrointestinal stromal tumors and colon carcinomas [34-36]. In agreement with this fact we found that HMGB1 was up-regulated in DLD1+7 cell clones with one of the highest fold-changes (2.01). Essentially, HMGB1 promotes cancer cells to metastasize [37] and various methods are published focusing on HMGB1 as a potential cancer therapy target [38-41]. HMGB1 protects DNA from damaging agents and thus guards against the generation of genomic aberrations $[42,43]$. This linkage between HMGB1, maintenance of genome stability and prevention of human diseases suggests HMGB1 up-regulation to be an important mechanism of the cell to maintain genomic instability. Notably, HMGB1 interacts with PSMB3 and IDH3A in the same overlapping networks (Table 1 and Fig. 2a). In contrast to HMGB1 and PCNA, IDH3A and PSMB3 were found to be significantly down-regulated in both, DLD1+7 and DLD1+13 clones. The IDH3A gene encodes an isocitrate dehydrogenase that generally catalyzes the oxidative decarboxylation of isocitrate to 2-oxoglutarate and therefore plays a role in intermediary metabolism and energy production. Isocitrate dehydrogenases belong to two distinct subclasses, one of which utilizes $\mathrm{NAD}(+)$ as the electron acceptor and the other NADP(+). PSMB3 encodes a member of the proteasome B-type family, also known as the T1B family, which is a $20 \mathrm{~S}$ core beta subunit [44]. Proteasomes are distributed throughout eukaryotic cells at a high concentration and cleave peptides in an ATP/ubiquitin-dependent process in a non-lysosomal pathway. However, IDH3A and PSMB3 has to be functionally characterize for their involvement in regulation of genomic stability.

Another important finding of our results is that chromosomal aneuploidy leads to up-regulation of growth-promoting genes and down-regulation of genes involved in growth control. We show that chromosomes not observed to be aneuploid in particular tumor types (i.e., chromosome 3 in colorectal tumors) have an increased activity regarding the translation into proteins. Thus, the presence of a trisomy is not neutral with respect to the proteome / pathways and could be the results of a selective pressure against maintaining extra copies of chromosome 3. This finding is supported by the fact that aneuploidy due to the gain of a single chromosome can indeed result in deregulation of 100-200 genes [9] and the reversion of tumor pheno- types upon microcell-mediated chromosome transfers $[45,46]$. On the other hand, for trisomic chromosomes playing an important role during colorectal tumorigenesis, i.e., for trisomy 7 , the earliest detectable chromosomal alteration at pre-malignant stages [6], we find significant protein pattern of decreased structural molecule activity but increased catalytic, translational, and ion channel activity that differs from chromosomes not at all, or at a later stage, involved in colorectal tumor development (Fig. 3). Especially, the translation process of key regulatory initiation factors have been documented in various steps of cancer development including migration, invasion, and angiogenesis $[47,48]$. In this context, we identified the transcription factor EIF3I known to promote colon oncogenesis by the regulation of COX-2 and $\beta$-catenin [49]. We conclude that cells harboring an extra copy of chromosome 7 seem to be equipped with a competitive growth and proliferation advantage, key features needed for colorectal adenoma development and malignant transformation. Further functional experiments against the background of a positive expression of certain oncogenes in the parental cell line are warranted.

\section{Conclusions}

The trisomies now generated affected the expression of 79 proteins that do not map on the trisomic chromosome but are involved in the regulation of genomic stability, e.g. PCNA, HMGB1, IDH3A, and PSMB3. These changes of protein expression patterns reflect a substantial, pathway-related alteration of the cellular equilibrium. Particularly trisomy-7, the earliest chromosomal aberration in premalignant stages of colorectal carcinomas, is associated with protein expression changes that affect molecular functions involved in cell growth and proliferation.

\section{Acknowledgments}

Grants from the Swedish Cancer Society, the Cancer Society Stockholm, the Swedish Research Council, King Gustav V Jubilee Fund, Wallenberg Consortium North, Knut and Alice Wallenberg Foundation, Werner and Clara Kreitz Foundation and the Ad Infinitum Foundation are gratefully acknowledged. This study was performed in connection with the Surgical Center for Translational Oncology - Lübeck (SCTO-L) 
and the North German Tumorbank of Colorectal Cancer (ColoNet), the latter being generously supported by the German Cancer Aid Foundation (DKH e.V. \# 108446).

\section{Conflict of interests}

All authors declare that they have no competing financial interest.

\section{Additional files}

File name: Additional file 1

File format: PDF

Title: $\quad$ Microcell-mediated Chromosome-

Transfer

Description: Detailed methodical description of the microcell-mediated chromosometransfer.

File name: Additional file 2

File format: PDF

Title: $\quad$ In-gel digestion and mass spectrometry (MALDI-ToF-MS)

Description: Detailed methodical description for the applied in-gel digestion and mass spectrometry by MALDI-ToF-MS.

File name: Additional file 3

File format: PDF

Title: $\quad$ Identifications of proteins of the DLD1 versus DLD1+3 analysis

Description: Detailed list of all identified proteins of the DLD1 versus DLD1+3 comparison.

File name: Additional file 4

File format: PDF

Title: Overview of signaling networks with highest scores

Description: Detailed list of all networks with highest scores of all pair-wise group comparisons.

File name: Additional file 5

File format: PDF

Title: $\quad$ IPA analysis of the DLD1 versus

DLD1+3 comparison

Description: IPA gene pathway associated with Lipid Metabolism, Molecular Transport, and Small Molecule Biochemistry in the DLD1+3 comparison.
File name: Additional file 6

File format: PDF

Title: Gel images

Description: Representative gel image of DLD1, DLD1+3, DLD1+7, DLD1+13 cell clones and one example of an up- and a down-regulated protein, respectively.

\section{References}

[1] T.O. Caspersson, Cancer Res 39 (1979), 2341-2345.

[2] E.M. Torres, B.R. Williams and A. Amon, Genetics 179 (2008), 737-746.

[3] B.R. Williams and A. Amon, Cancer Research 69 (2009), 5289-5291.

[4] A.J. Holland and D.W. Cleveland, Nat Rev Mol Cell Biol 10 (2009), 478-487.

[5] T. Gemoll, U.J. Roblick, S. Szymczak, T. Braunschweig, S. Becker, B.W. Igl, H.P. Bruch, A. Ziegler, U. Hellman, M.J. Difilippantonio, T. Ried, H. Jornvall, G. Auer and J.K. Habermann, Cell Mol Life Sci 68 (2011), 3261-3274.

[6] L. Bomme, G. Bardi, N. Pandis, C. Fenger, O. Kronborg and S. Heim, Genes Chromosomes Cancer 10 (1994), 190-196.

[7] T. Ried, R. Knutzen, R. Steinbeck, H. Blegen, E. Schrock, K. Heselmeyer, S. du Manoir and G. Auer, Genes Chromosomes Cancer 15 (1996), 234-245.

[8] J.K. Habermann, U. Paulsen, U.J. Roblick, M.B. Upender, L.M. McShane, E.L. Korn, D. Wangsa, S. Kruger, M. Duchrow, H.P. Bruch, G. Auer and T. Ried, Genes Chromosomes Cancer 46 (2007), 10-26.

[9] M.B. Upender, J.K. Habermann, L.M. McShane, E.L. Korn, J.C. Barrett, M.J. Difilippantonio and T. Ried, Cancer Research 64 (2004), 6941-6949.

[10] L.V. Leak, L.A. Liotta, H. Krutzsch, M. Jones, V.A. Fusaro, S.J. Ross, Y. Zhao and E.F. Petricoin, 3rd, Proteomics 4 (2004), 753-765.

[11] U.J. Roblick, D. Hirschberg, J.K. Habermann, C. Palmberg, S. Becker, S. Kruger, M. Gustafsson, H.P. Bruch, B. Franzen, T. Ried, T. Bergmann, G. Auer and H. Jornvall, Cell Mol Life Sci 61 (2004), 1246-1255.

[12] R.E. Fournier and J.A. Frelinger, Mol Cell Biol 2 (1982), 526-534.

[13] P.J. Saxon and E.J. Stanbridge, Methods Enzymol 151 (1987), 313-325.

[14] M.M. Bradford, Analytical Biochemistry 72 (1976), 248-254.

[15] J.M. Bland and D.G. Altman, Lancet 1 (1986), 307-310.

[16] U. Hellman, EXS 88 (2000), 43-54.

[17] H. Mi, Q. Dong, A. Muruganujan, P. Gaudet, S. Lewis and P.D. Thomas, Nucleic Acids Res 38 (2010), D204-D210.

[18] K. Sengupta, M.B. Upender, L. Barenboim-Stapleton, Q.T. Nguyen, S.M. Wincovitch, Sr., S.H. Garfield, M.J. Difilippantonio and T. Ried, PLoS One 2 (2007), e199.

[19] N.D. Perkins, Oncogene 25 (2006), 6717-6730.

[20] B.M. Kessler and M.J. Edelmann, Cell Biochem Biophys 60 (2011), 21-38.

[21] S. Stingele, G. Stoehr, K. Peplowska, J. Cox, M. Mann and Z. Storchova, Molecular Systems Biology 8 (2012), 608. 
[22] E.M. Torres, N. Dephoure, A. Panneerselvam, C.M. Tucker, C.A. Whittaker, S.P. Gygi, M.J. Dunham and A. Amon, Cell 143 (2010), 71-83.

[23] T.S. Krishna, X.P. Kong, S. Gary, P.M. Burgers and J. Kuriyan, Cell 79 (1994), 1233-1243.

[24] T. Tsurimoto, Biochim Biophys Acta 1443 (1998), 23-39.

[25] D.J. Mozzherin, M. McConnell, M.V. Jasko, A.A. Krayevsky, C.K. Tan, K.M. Downey and P.A. Fisher, J Biol Chem 271 (1996), 31711-31717.

[26] R. Gary, D.L. Ludwig, H.L. Cornelius, M.A. MacInnes and M.S. Park, J Biol Chem 272 (1997), 24522-24529.

[27] R.E. Johnson, G.K. Kovvali, S.N. Guzder, N.S. Amin, C. Holm, Y. Habraken, P. Sung, L. Prakash and S. Prakash, J Biol Chem 271 (1996), 27987-27990.

[28] A. Umar, A.B. Buermeyer, J.A. Simon, D.C. Thomas, A.B. Clark, R.M. Liskay and T.A. Kunkel, Cell 87 (1996), 65-73.

[29] S.J. Muller-Weeks and S. Caradonna, Exp Cell Res 226 (1996), 346-355.

[30] X. Li, J. Li, J. Harrington, M.R. Lieber and P.M. Burgers, J Biol Chem 270 (1995), 22109-22112.

[31] Z. Kelman and J. Hurwitz, Trends Biochem Sci 23 (1998), 236-238.

[32] N. Mailand, I. Gibbs-Seymour and S. Bekker-Jensen, Nat Rev Mol Cell Biol 14 (2013), 269-282.

[33] M. Stros, Biochimica et Biophysica Acta 1799 (2010), 101-113.

[34] L. Campana, L. Bosurgi and P. Rovere-Querini, Curr Opin Immunol 20 (2008), 518-523.

[35] K. Volp, M.L. Brezniceanu, S. Bosser, T. Brabletz, T. Kirchner, D. Gottel, S. Joos and M. Zornig, Gut 55 (2006), 234-242.

[36] M.L. Brezniceanu, K. Volp, S. Bosser, C. Solbach, P. Lichter, S. Joos and M. Zornig, Faseb J 17 (2003), 1295-1297.
[37] H. Ohmori, Y. Luo and H. Kuniyasu, Expert Opin Ther Targets 15 (2011), 183-193.

[38] H. Ohmori, Y. Luo, K. Fujii, T. Sasahira, T. Shimomoto, A. Denda and H. Kuniyasu, Pathobiology 77 (2010), 210-217.

[39] H. Kuniyasu, N. Oue, A. Wakikawa, H. Shigeishi, N. Matsutani, K. Kuraoka, R. Ito, H. Yokozaki and W. Yasui, J Pathol 196 (2002), 163-170.

[40] K. Fujii, Y. Luo, T. Sasahira, A. Denda, H. Ohmori and H. Kuniyasu, Cell Prolif 42 (2009), 701-709.

[41] J. Xu, M. Futakuchi, M. Iigo, K. Fukamachi, D.B. Alexander, H. Shimizu, Y. Sakai, S. Tamano, F. Furukawa, T. Uchino, H. Tokunaga, T. Nishimura, A. Hirose, J. Kanno and H. Tsuda, Carcinogenesis 31 (2010), 927-935.

[42] S. Giavara, E. Kosmidou, M.P. Hande, M.E. Bianchi, A. Morgan, F. d'Adda di Fagagna and S.P. Jackson, Curr Biol 15 (2005), 68-72.

[43] Y. Liu, R. Prasad and S.H. Wilson, Biochimica Et Biophysica Acta 1799 (2010), 119-130.

[44] K. Tanaka, T. Tamura, T. Yoshimura and A. Ichihara, New Biol 4 (1992), 173-187.

[45] X. Yang, Q. Tahin, Y.F. Hu, I.H. Russo, B.R. Balsara, D. Mihaila, C. Slater, J.C. Barrett and J. Russo, International Journal of Oncology 15 (1999), 629-638.

[46] M. Wan, T. Sun, R. Vyas, J. Zheng, E. Granada and L. Dubeau, Oncogene 18 (1999), 1545-1551.

[47] C.G. Proud, European Journal of Biochemistry/FEBS 269 (2002), 5337.

[48] M.J. Clemens, Oncogene 23 (2004), 3180-3188.

[49] J. Qi, Z. Dong, J. Liu and J.T. Zhang, Oncogene (2013). 


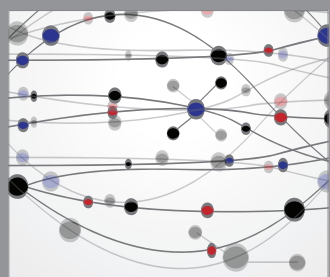

The Scientific World Journal
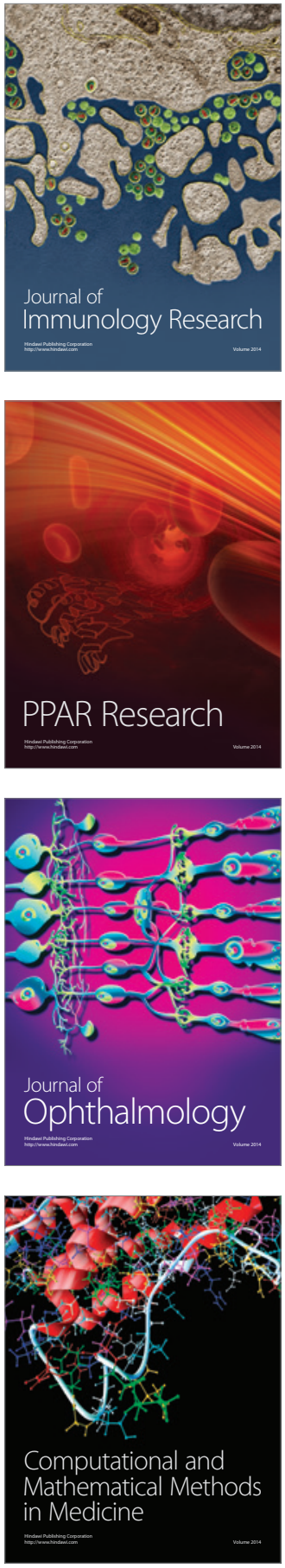

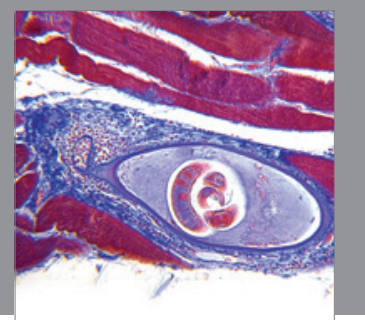

Gastroenterology

Research and Practice
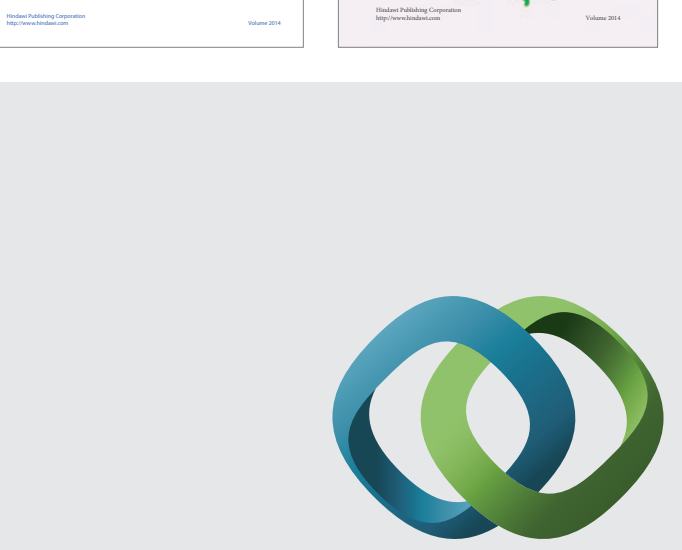

\section{Hindawi}

Submit your manuscripts at

http://www.hindawi.com
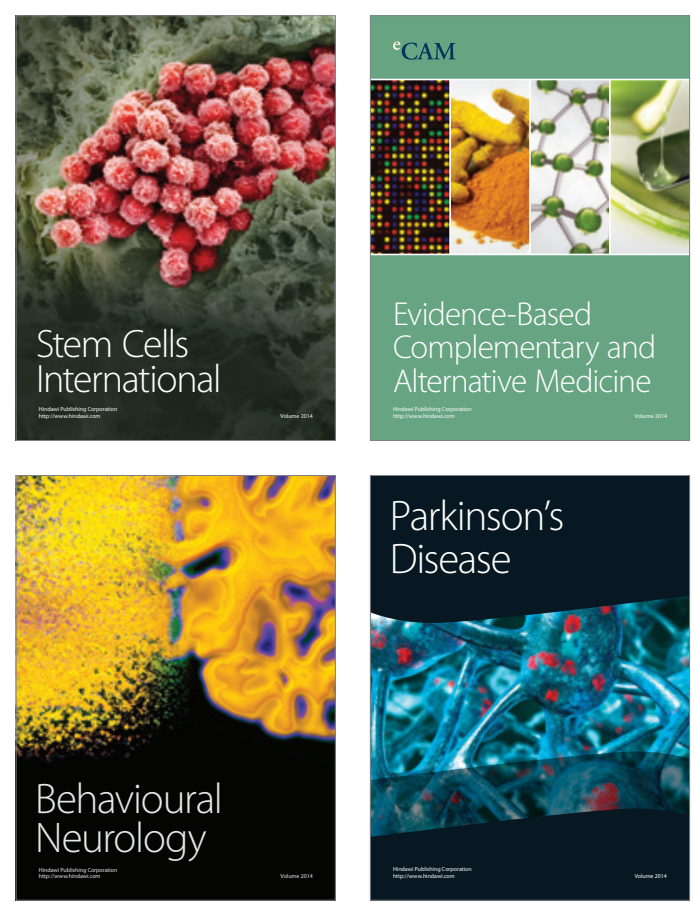

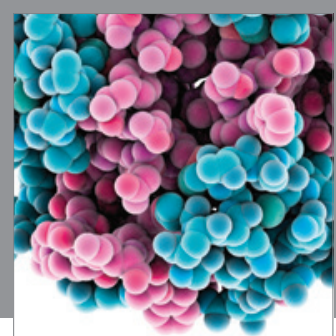

Journal of
Diabetes Research

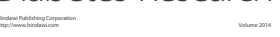

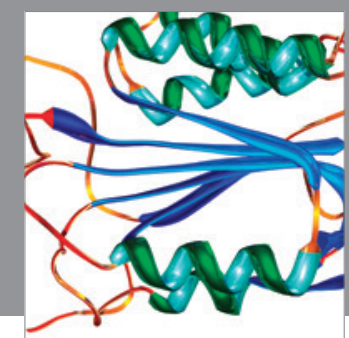

Disease Markers
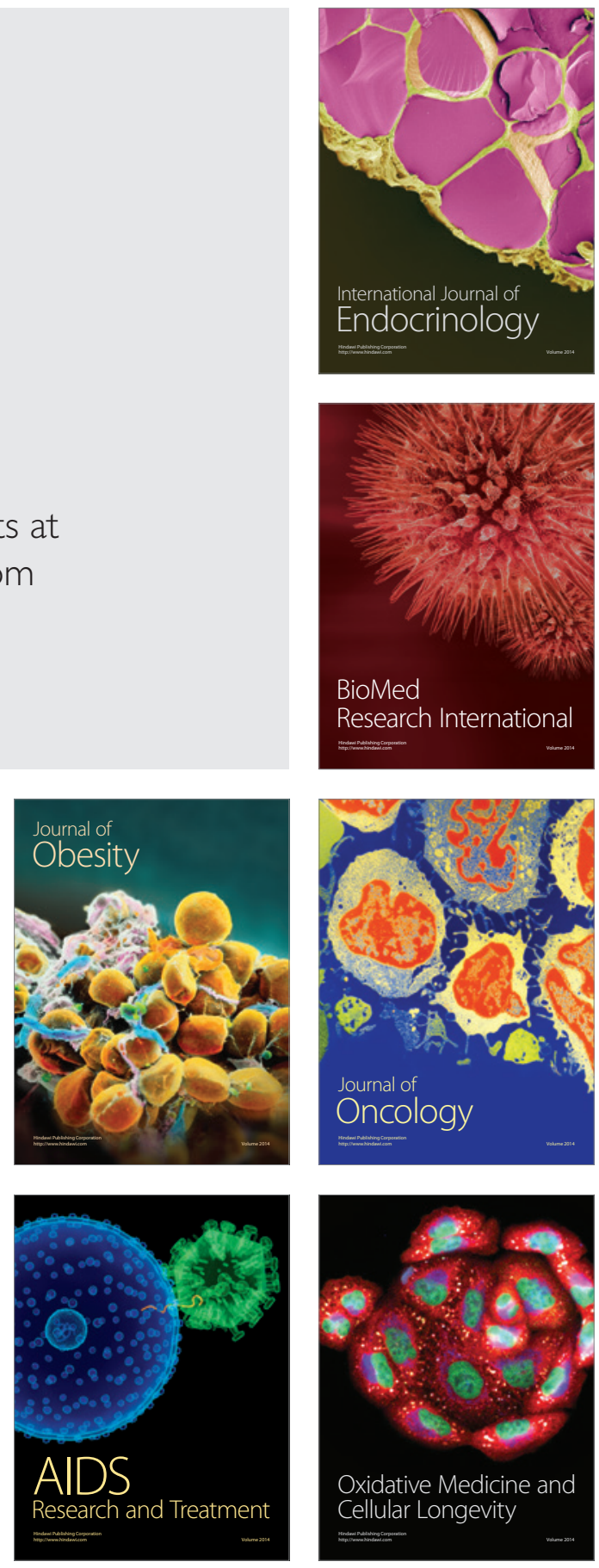